Article

\title{
Capparis spinosa Fruit Ethanol Extracts Exert Different Effects on the Maturation of Dendritic Cells
}

\author{
Azeguli Hamuti ${ }^{1}$, Jinyu Li ${ }^{1}$, Fangfang Zhou ${ }^{1}$, Adila Aipire ${ }^{1}$, Ji Ma ${ }^{1}$, Jianhua Yang ${ }^{1,2}$ and \\ Jinyao $\mathrm{Li}^{1, *}$ \\ 1 Xinjiang Key Laboratory of Biological Resources and Genetic Engineering, \\ College of Life Science and Technology, Xinjiang University, 666 Shengli Road, Urumqi 830046, China; \\ 13070411128@163.com (A.H.); lijinyu234@163.com (J.L.); 15199131636@163.com (F.Z.); \\ kaskas999@163.com (A.A.); majiuci@xju.edu.cn (J.M.); jianhuay@bcm.edu (J.Y.) \\ 2 Texas Children's Cancer Center, Department of Pediatrics, Dan L. Duncan Cancer Center, \\ Baylor College of Medicine, Houston, TX 77030, USA \\ * Correspondence: ljyxju@xju.edu.cn; Tel.: +86-991-8583259; Fax: +86-991-8583517 \\ Academic Editor: Chun Kwok Wong \\ Received: 29 November 2016; Accepted: 2 January 2017; Published: 7 January 2017
}

\begin{abstract}
Capparis spinosa L. (C. spinosa) has been used as food and traditional medicine and shows anti-inflammatory and anti-oxidant activities. Here, we prepared the $C$. spinosa fruit ethanol extracts (CSEs) using different procedures and investigated the effects of CSE on the maturation of mouse bone marrow-derived dendritic cells (DCs) in the absence or presence of lipopolysaccharide (LPS). DC maturation and cytokine production were detected by flow cytometry and ELISA, respectively. We obtained three different CSEs and dissolved in water or DMSO, named CSE2W, CSEMW, CSE3W, CSE2D, CSEMD, and CSE3D, respectively. These CSEs showed different effects on DC maturation. CSEMW and CSEMD significantly increased the expressions of CD40, CD80, and CD86, in a dose-dependent manner. CSE2W and CSE2D also showed a modest effect on DC maturation, which enhanced the expression of CD40. CSE3W and CSE3D did not change DC maturation but suppressed LPS-induced DC maturation characterized by the decreased levels of CD40 and CD80. CSE3W and CSE3D also significantly inhibited the secretions of IL-12p40, IL-6, IL-1 $\beta$, and TNF- $\alpha$ induced by LPS. CSE3W further increased the level of IL-10 induced by LPS. Moreover, CSE3D suppressed LPS-induced DC maturation in vivo, which decreased the expressions of CD40 and CD80. These results suggested that CSE3W and CSE3D might be used to treat inflammatory diseases.
\end{abstract}

Keywords: Capparis spinosa; dendritic cell; maturation; cytokine production

\section{Introduction}

Traditional Chinese medicines (TCM) have been used to treat various kinds of diseases for a long history. Accumulating evidence has shown that TCM exerts immunoregulatory effects to prevent or treat infection or autoimmune diseases [1,2]. TCM could promote the maturation of dendritic cells (DCs) to enhance immune responses against infection, or suppress DC maturation to inhibit immune responses against inflammation or autoimmune diseases [1,3]. Capparis spinosa $\mathrm{L}$. is a kind of TCM and has been used to treat gastro-intestinal problems, rheumatism, gout, diabetes, inflammation, kidney, and liver diseases [4-7]. In China, C. spinosa (also named wild watermelon) mainly distributed in Xinjiang Uygur Autonomous Region, which is a kind of sand binding plant and its buds, leaves, roots, and fruits have been used in traditional Uighur Medicine to treat gout and rheumatoid arthritis for a long time [8]. Recently, many studies have been reported that C. spinosa contains a lot of biochemical compounds including flavonoids, alkaloids, polyphenols, saponins, terpenoids, lectin, essential oils, glycosinolate, and glycosides [6,9-12], which exhibited a broad range of activities such as anti-inflammatory [13-16], anti-viral [17,18], anti-allergeric [19], 
anti-arthritic [20], anti-tumor [9,17,21], anti-oxidant [22-26], anti-nociceptive [27,28], anti-diabetic [4,29], anti-hepatotoxic [30], anti-hyperglycemic [31], hypolipidemic [32], and immunomodulatory [18,33]. However, the effect of $C$. spinosa on the maturation and cytokine production of DCs is still elusive.

DCs are professional antigen presenting cells and play a pivotal role in the immune system, which link the innate and adaptive immune responses. The activation status of DCs, including maturation and cytokine secretion, determined the development of $\mathrm{CD}^{+}$helper $\mathrm{T}(\mathrm{Th})$ cell subsets that helped $\mathrm{CD}^{+} \mathrm{T}$ cells to generate cytotoxic $\mathrm{T}$ lymphocytes and $\mathrm{B}$ cells to produce antibodies $[34,35]$. Therefore, immune responses including inflammation could be modulated through the regulation of DC activation status. In order to investigate whether $C$. spinosa can affect the DC activation status to exert its some pharmacological effects, such as anti-inflammatory, anti-allergic, and immunomodulatory, we prepared C. spinosa fruit ethanol extracts (CSEs) and detected their effects on the maturation and cytokine production of DCs, especially in the presence of lipopolysaccharide (LPS).

\section{Results}

\subsection{The Preparation of CSEs and Their Effect on DC Viability}

CSEs were prepared using ethanol extract following the extraction with petroleum ether and ethyl acetate according to previous description [36]. To separate the components with inhibitory activity from other components with stimulative activity on DC maturation, we optimized the procedures through changing the content of ethanol during evaporation and adding $\mathrm{NaCl}$ after petroleum ether extraction (Figure 1). We collected these fractions and named them CSE2, CSEM, and CSE3. These CSEs were dissolved in distilled water or DMSO at $200 \mathrm{mg} / \mathrm{mL}$ and named CSE2W, CSEMW, and CSE3W; or CSE2D, CSEMD and CSE3D, respectively. The contents of flavonoids and polysaccharides were determined by AlCl3-KAC and anthrone-sulfuric acid method [37,38], respectively. The concentrations of polysaccharides and flavonoids in CSEs were shown in Table 1.

To detect the effect of CSEs on DC viability, different concentrations of CSE2W, CSEMW, and CSE3W according to their polysaccharide contents $(0.17,0.85$, and $1.7 \mathrm{mg} / \mathrm{mL})$ or CSE2D, CSEMD, and CSE3D according to their flavonoid contents $(1.2,1.8$, and $2.4 \mu \mathrm{g} / \mathrm{mL})$ were used to treat DCs. After $12 \mathrm{~h}$, DCs were collected and stained with PI/Annexin V kit. Samples were analyzed by flow cytometry. The results showed that all CSEs in selected concentrations did not significantly change the frequencies of necrotic $\left(\mathrm{PI}^{+}\right.$Annexin $\mathrm{V}^{-}$) and apoptotic $\left(\mathrm{PI}^{-}\right.$Annexin $\mathrm{V}^{+}$and $\mathrm{PI}^{+}$Annexin $\left.\mathrm{V}^{+}\right) \mathrm{DCs}$ (Figure 2), suggesting that CSEs did not dampen the viability of DCs. 


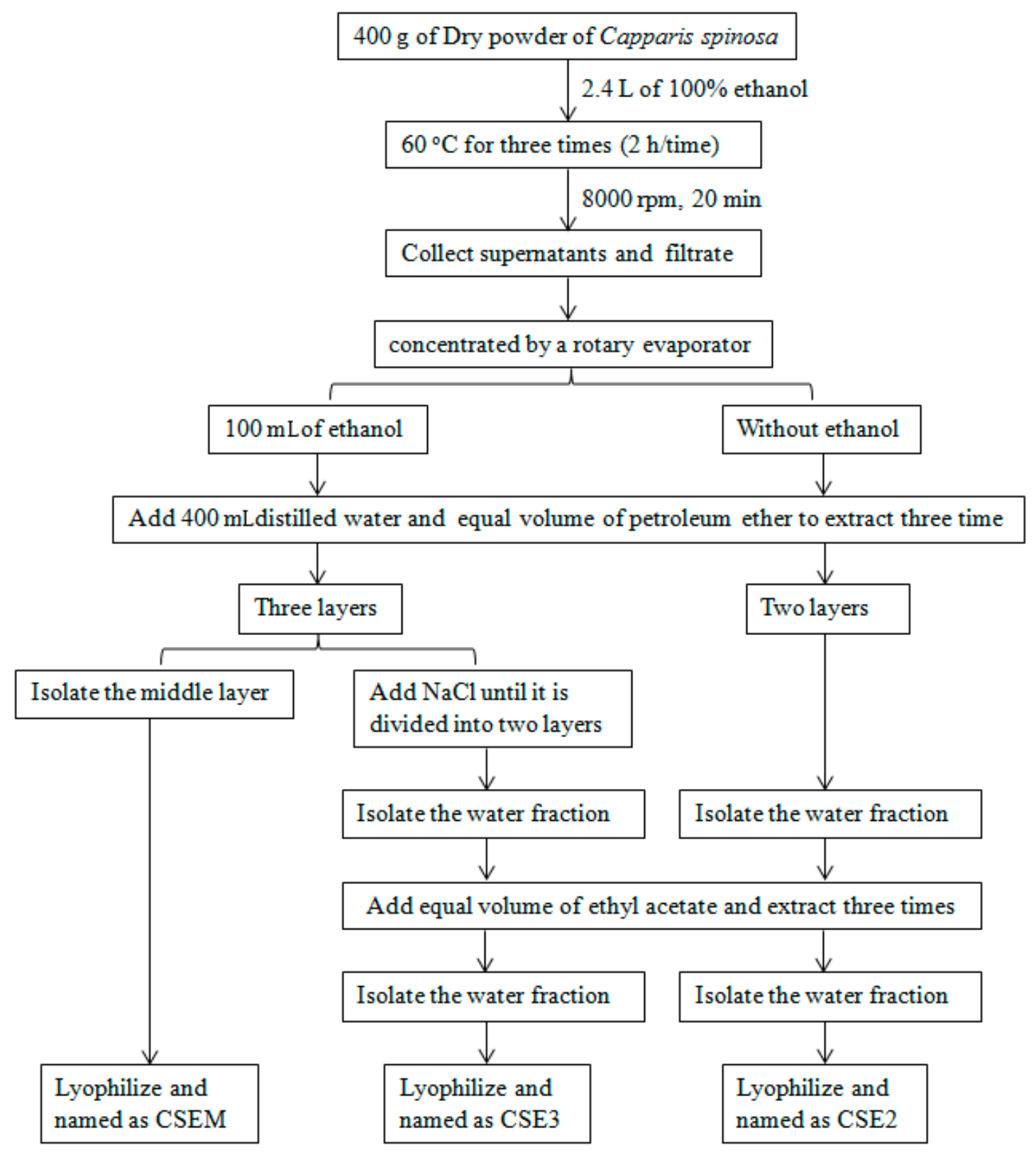

Figure 1. The extraction procedures for CSE2, CSEM, and CSE3.

Table 1. Contents of flavonoids and polysaccharides in Capparis spinosa extracts.

\begin{tabular}{cccc}
\hline Extracts & $\begin{array}{c}\text { Polysaccharides } \\
(\mathbf{m g} / \mathbf{m L})\end{array}$ & $\begin{array}{c}\text { Flavonoids } \\
\mathbf{( m g / m L )}\end{array}$ & $\begin{array}{c}\text { Ratios of } \\
\text { Polysaccharides/Flavonoids }\end{array}$ \\
\hline CSE2W & 115.58 & 0.34 & 340 \\
CSE2D & 97.87 & 0.26 & 376 \\
CSEMW & 67.17 & 0.46 & 146 \\
CSEMD & 66.91 & 0.79 & 85 \\
CSE3W & 133.23 & 0.2 & 666 \\
CSE3D & 58.81 & 0.16 & 368 \\
\hline
\end{tabular}



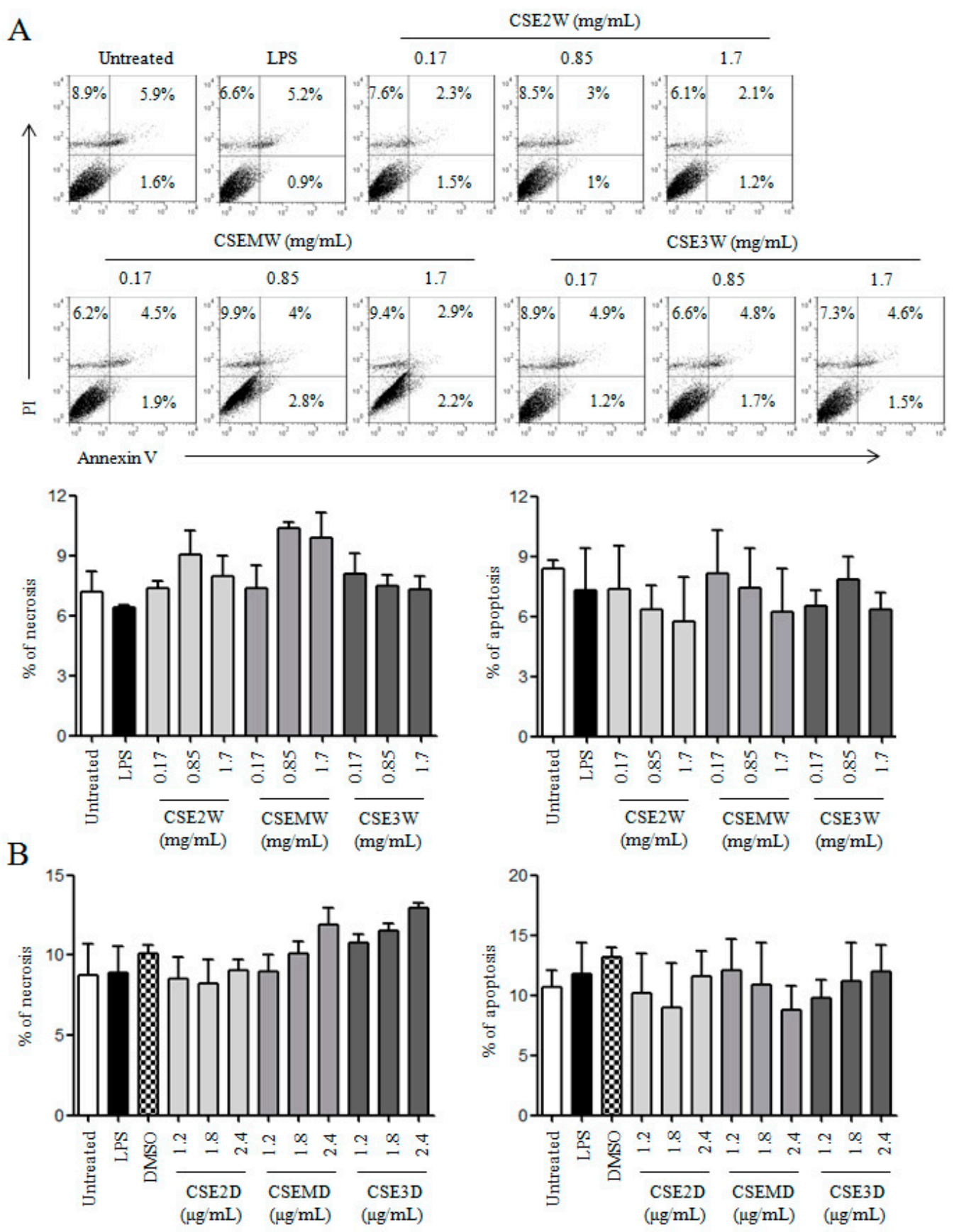

Figure 2. The effect of CSEs on DC viability. Mouse bone marrow-derived DCs were treated with different concentrations of polysaccharides in CSE2W, CSEMW, and CSE3W (A) or flavonoids in CSE2D, CSEMD, and CSE3D (B). After $12 \mathrm{~h}$, cells were collected and stained with Annexin V/PI kit. The representative dot plots are shown in upper panels. The summary of necrotic and apoptotic DCs are shown in lower panels.

\subsection{The Effect of Polysaccharides in CSEs on DC Maturation and Cytokine Production}

Several studies including ours have been reported that polysaccharides from herbal medicines can promote DC maturation and cytokine production [39-42]. The effect of polysaccharides in CSE2W, CSEMW, and CSE3W on DC maturation was investigated. After treatment with different polysaccharide concentrations $(0.17,0.85$, and $1.7 \mathrm{mg} / \mathrm{mL})$ of CSEs, the expressions of CD40, CD80, CD86, and MHC II on DCs were detected by flow cytometry (Figure 3A). We observed that CSEMW significantly increased the expressions of CD40, CD80, and CD86, in a dose-dependent manner, which 
is even higher than that induced by LPS. The highest and middle dose of CSE2W also significantly increased the expressions of CD40 and CD80, respectively, although the level is dramatically lower than that induced by CSEMW. However, CSE3W did not increase the expressions of all these molecules. The levels of IL-12p40, TNF- $\alpha$, and IL-10 in supernatants of the above samples were measured by ELISA. As shown in Figure 3B, LPS induced high levels of IL-12p40 and TNF- $\alpha$ but all CSEs dissolved in water did not increase the secretions of IL-12p40 and TNF- $\alpha$. Compared to the untreated group, the level of IL-10 had no significant change upon the treatment of CSEs. These results indicate that polysaccharides in CSEs have different effects on DC maturation.

A
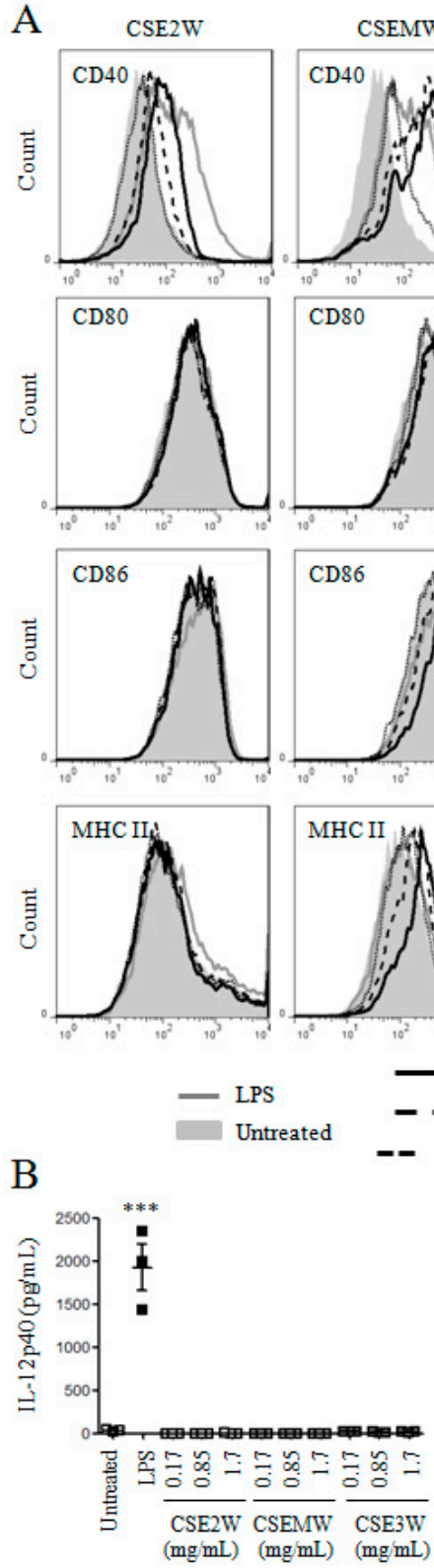
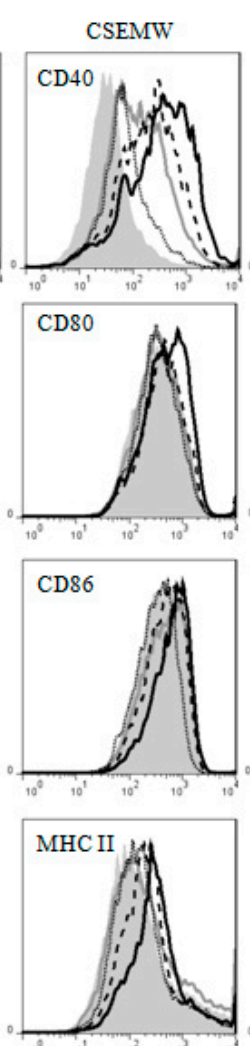

$\left.\begin{array}{r}1.7 \\ -\cdot 0.85 \\ -\quad 0.17\end{array}\right]$

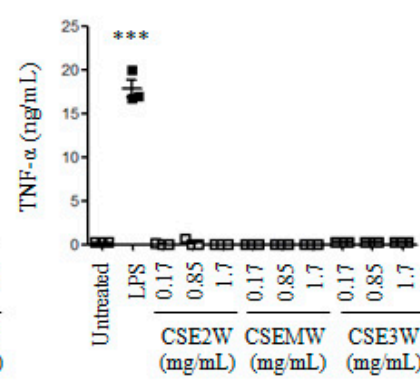

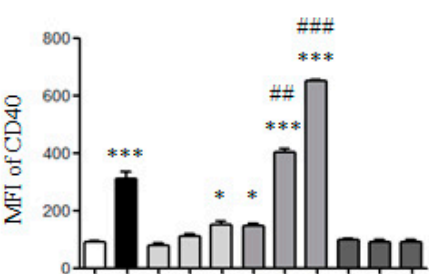
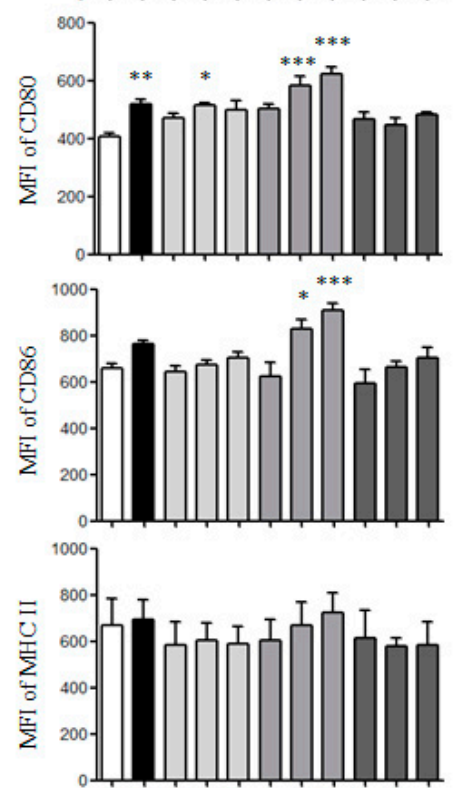

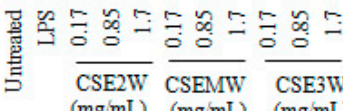

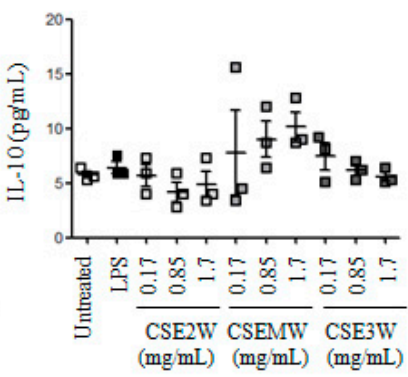

Figure 3. The effect of polysaccharides in CSE2W, CSEMW, and CSE3W on DC maturation. DCs were treated with different concentrations of polysaccharides in CSE2W, CSEMW, and CSE3W for $12 \mathrm{~h}$. (A) Cells were collected to analyze the expressions of CD40, CD80, CD86, and MHC II by flow cytometry. MFI of these molecules is shown; (B) The supernatants were collected to detect the secretions of IL-12p40, IL-10, and TNF- $\alpha$ by ELISA. The concentrations of IL-12p40, IL-10, and TNF- $\alpha$ are shown. Data are from four independent experiments and analyzed by ANOVA. ${ }^{*} p<0.05 ;{ }^{* *} p<0.01$; *** $p<0.001$ compared to untreated DCs. \#\# $p<0.01$; \#\#\# $p<0.001$ compared to LPS treated DCs. 


\subsection{The Effect of Flavonoids in CSEs on DC Maturation and Cytokine Production}

It has been shown that flavonoids in C. spinosa have anti-inflammatory activity $[14,16]$. Therefore, we detected the effect of flavonoids in CSEs on DC maturation and cytokine production. Firstly, different flavonoid concentrations $(1.2,1.8$, and $2.4 \mu \mathrm{g} / \mathrm{mL})$ of CSE2W, CSEMW, and CSE3W were used to treat DCs. After $12 \mathrm{~h}$, the expressions of CD40 and CD80 on DCs were measured by flow cytometry. Similar with Figure 3A, CSEMW dose-dependently enhanced the expressions of CD40 and CD80 and the stimulatory activity was higher than that of LPS. CSE2W also significantly enhanced the expression of CD40 but not for CD80. CSE3W still did not enhance the expressions of these molecules (Figure 4A). Secondly, the same flavonoid concentrations of CSE2D, CSEMD, and CSE3D were used to treat DCs for $12 \mathrm{~h}$. Similar results were obtained compared with CSE2W, CSEMW, and CSE3W contained the same concentrations of flavonoids (Figure 4B). All CSEs dissolved in DMSO did not increase the production of IL-12p40, TNF- $\alpha$, and IL-10 (Figure 4C).
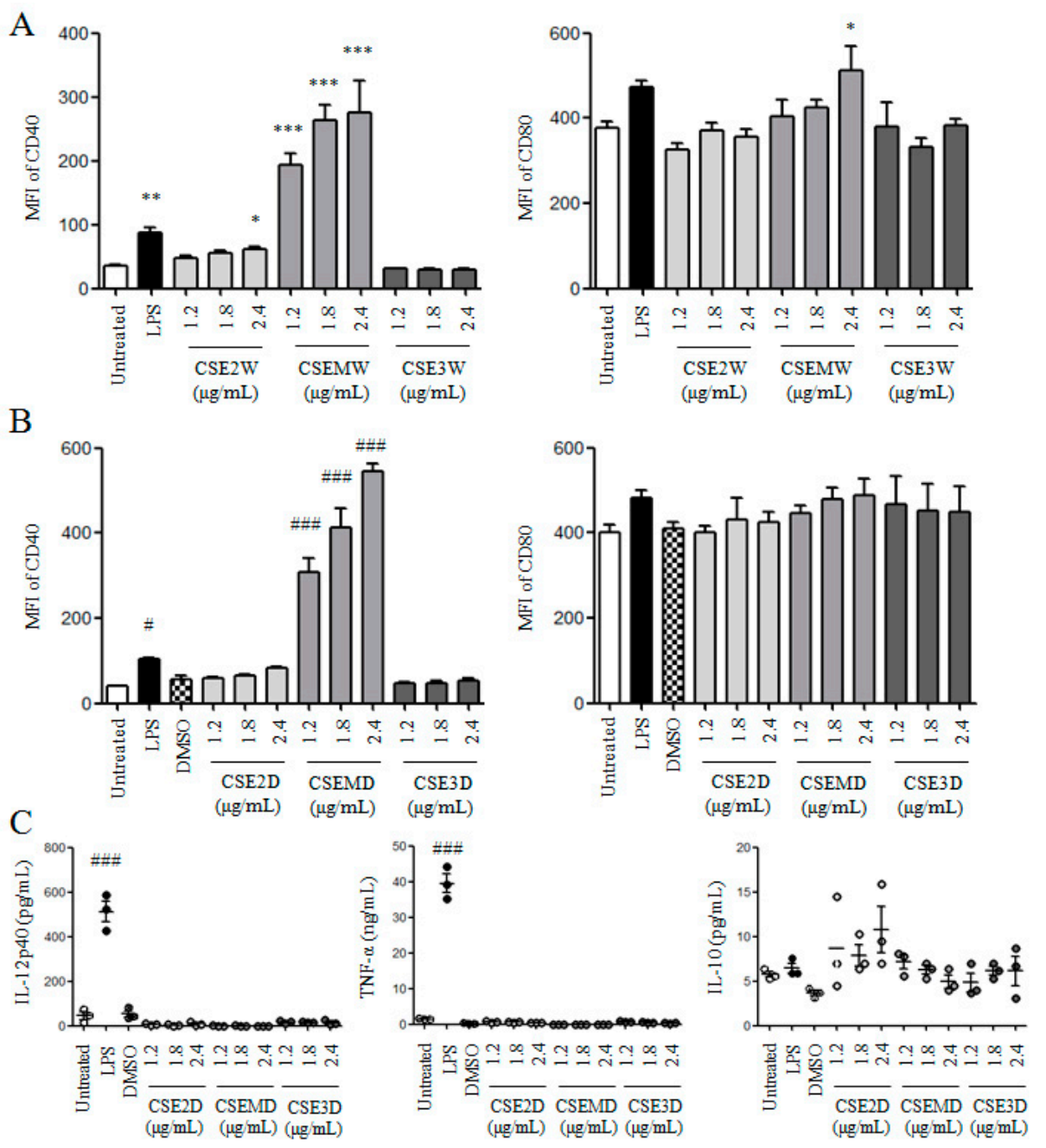

Figure 4. The effect of flavonoids in CSEs on DC maturation. DCs were treated with different concentrations of flavonoids in CSEs for $12 \mathrm{~h}$. Cells were collected to analyze the expressions of CD40 and CD80 by flow cytometry. (A) MFI of CD40 and CD80 upon CSE2W, CSEMW and CSE3W treatment; (B) MFI of CD40 and CD80 upon CSE2D, CSEMD, and CSE3D treatment; (C) The supernatants were collected to detect the levels of IL-12p40, IL-10, and TNF- $\alpha$ by ELISA after CSE2D, CSEMD, and CSE3D treatment. The concentrations of IL-12p40, IL-10, and TNF- $\alpha$ are shown. Data are from four independent experiments and analyzed by ANOVA. ${ }^{*} p<0.05 ;{ }^{* *} p<0.01$; ${ }^{* *} p<0.001$ compared to untreated DCs. \# $p<0.05$; \#\#\# $p<0.001$ compared to DMSO treated DCs. 


\subsection{CSE3D and CSE3W Suppressed DC Maturation and Cytokine Production Induced by LPS}

The above results showed that both CSE3D and CSE3W did not promote DC maturation and cytokine production. We wondered whether CSE3D and CSE3W could suppress DC maturation induced by LPS. DCs were treated with different flavonoid concentrations $(1.2,1.8$, and $2.4 \mu \mathrm{g} / \mathrm{mL})$ of CSE3D and CSE3W in the presence of LPS. After $12 \mathrm{~h}$, the stained samples were analyzed by flow cytometry. The results showed that both CSE3D and CSE3W significantly suppressed the expression of CD40 induced by LPS, in a dose-dependent manner. CSE3D also significantly suppressed the expression of CD80 induced by LPS but not for CSE3W (Figure 5A).

A
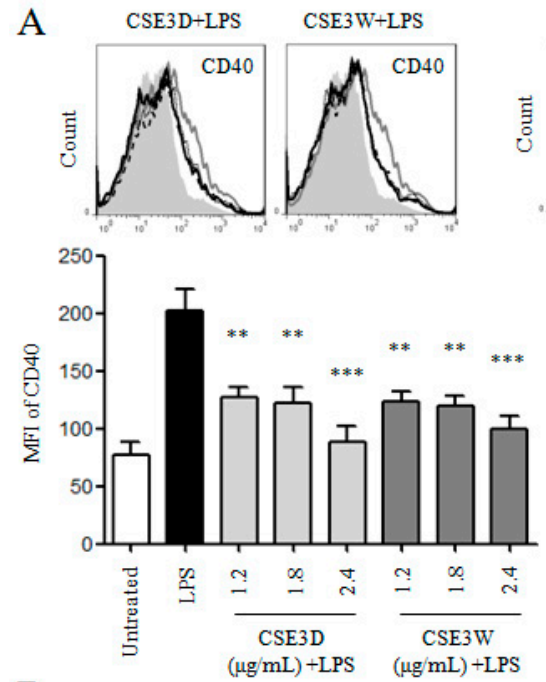
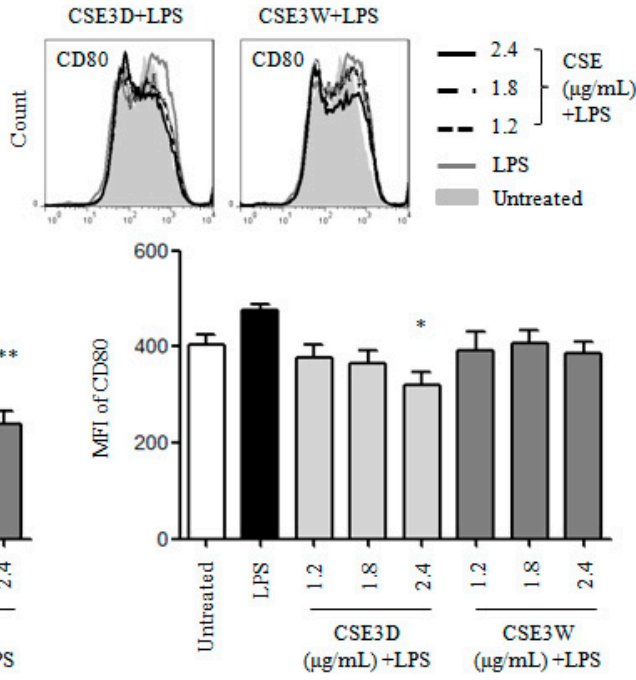

B
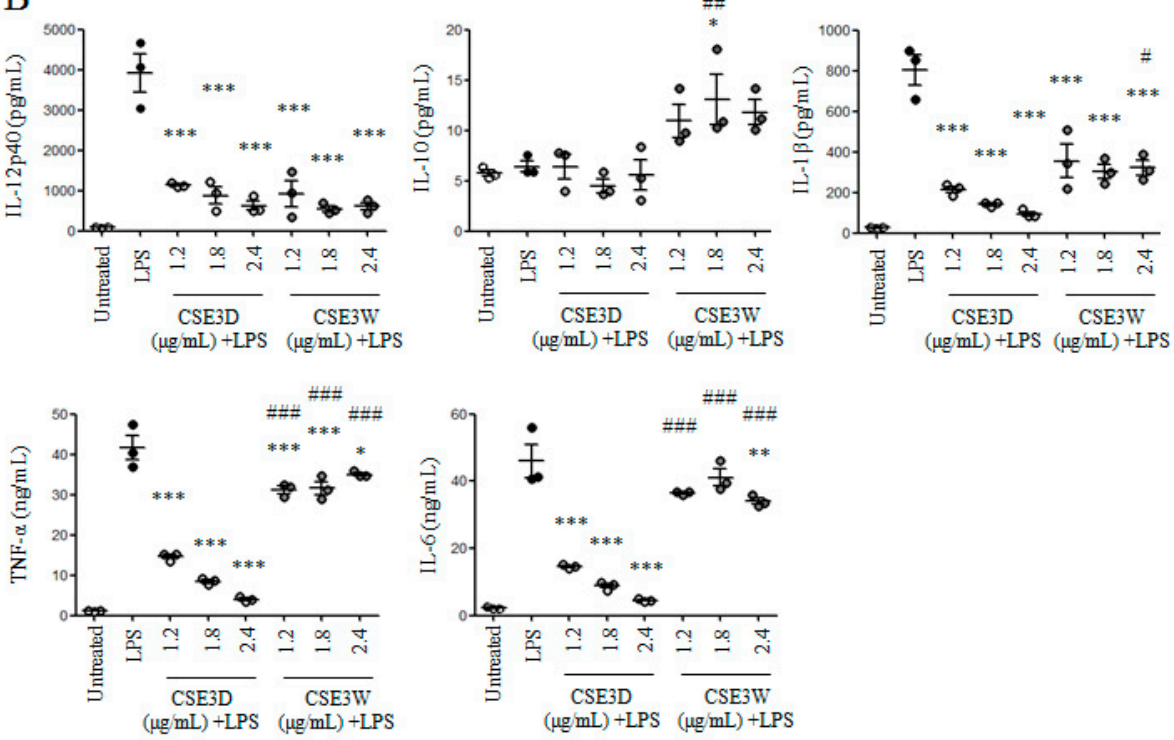

Figure 5. CSE3D and CSE3W suppressed DC maturation and cytokine production induced by LPS. DCs were treated with different concentrations of flavonoids in CSE3D and CSE3W in the presence of LPS for $12 \mathrm{~h}$. (A) Cells were collected to analyze the expressions of CD40 and CD80 by flow cytometry. MFI of CD40 and CD80 is shown; (B) The supernatants were collected to detect the levels of IL-12p40, IL-10, IL-6, IL-1 $\beta$, and TNF- $\alpha$ by ELISA. The concentrations of these cytokines are shown. Data are from three independent experiments and analyzed by ANOVA. ${ }^{*} p<0.05 ;{ }^{* *} p<0.01 ;{ }^{* * *} p<0.001$ compared to LPS treated DCs. \# $p<0.05$; \#\# $p<0.01$; \#\# $p<0.001$ compared to CSE3D + LPS treated DCs.

The levels of anti-inflammatory IL-10 and pro-inflammatory cytokines including IL-12p40, IL-6, IL- $1 \beta$, and TNF- $\alpha$ in supernatants of the above samples were determined by ELISA. We found that 
the levels of all these pro-inflammatory cytokines induced by LPS were significantly decreased by CSE3D and CSE3W (Figure 5B). Moreover, the inhibitory activity of CSE3D is stronger than that of CSE3W. However, the level of IL-10 induced by LPS was significantly increased by $1.8 \mu \mathrm{g} / \mathrm{mL}$ CSE3W. These results suggested that CSE3D and CSE3W could suppress DC maturation and pro-inflammatory cytokine production induced by LPS.

\subsection{CSE3D Suppressed DC Maturation Induced by LPS In Vivo}

The in vivo effect of CSE3D on DC maturation in the absence or presence of LPS was further investigated. BALB/c mice were injected with different flavonoid concentrations $(0.4,0.8$, and $1.6 \mathrm{\mu g} /$ mouse) of CSE3D by footpads. Mice injected with LPS alone were used as a positive control. After $24 \mathrm{~h}$, popliteal lymph nodes were isolated and lymphocytes were used to analyze the expressions of CD40, CD80, CD86, and MHC II on DCs $\left(\mathrm{CD} 11 \mathrm{c}^{+}\right)$by flow cytometry. The results showed that LPS significantly increased the expressions of CD40 and CD80 but CSE3D did not significantly change the expressions of all these molecules on DCs in vivo (Figure 6A).
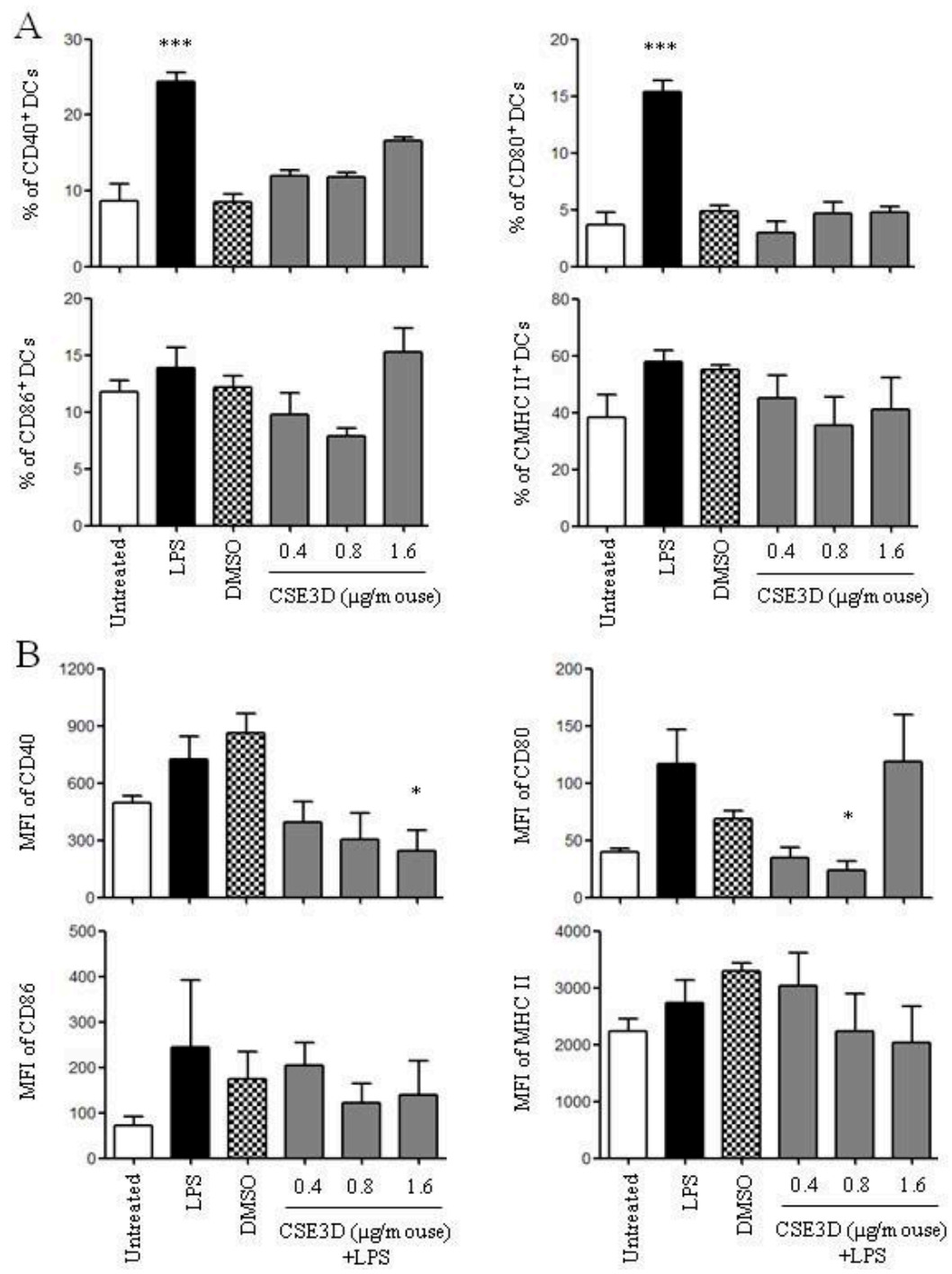

Figure 6. CSE3D suppressed DC maturation induced by LPS in vivo. Mice were injected with different concentrations of flavonoids in CSE3D in the absence (A) or presence (B) of LPS by footpads. After $24 \mathrm{~h}$, lymphocytes were isolated to analyze DC maturation. CD11 $\mathrm{c}^{+}$cells were gated to analyze the expressions of CD40, CD80, CD86, and MHC II. MFI of these molecules is shown. ${ }^{* * *} p<0.001$ compared to untreated mice. ${ }^{*} p<0.05$ compared to LPS treated mice. 
In order to detect the effect of CSE3D on DC maturation in vivo in the presence of LPS, mice were injected with different flavonoid concentrations $(0.4,0.8$, and $1.6 \mu \mathrm{g} /$ mouse) of CSE3D together with $100 \mathrm{ng} /$ mouse of LPS by footpads. After $24 \mathrm{~h}$, lymphocytes in popliteal lymph nodes were isolated to analyze DC maturation by flow cytometry. We observed that 1.6 and $0.8 \mu \mathrm{g} / \mathrm{mouse}$ of CSE3D significantly inhibited the expressions of CD40 and CD80 induced by LPS, respectively (Figure 6B). The expressions of CD86 and MHC II induced by LPS were also decreased although the difference was not significant. These results indicated that CSE3D could suppress DC maturation both in vitro and in vivo.

\section{Discussion}

We prepared the ethanol extracts of C. spinosa using different procedures and detected the effect of these extracts on DC maturation both in vitro and in vivo. We found that CSE2W, CSE2D, CSEMW, and CSEMD could promote DC maturation but CSE3W and CSE3D did not promote DC maturation. Interestingly, CSE3W and CSE3D suppressed DC maturation and pro-inflammatory cytokine production induced by LPS in vitro. CSE3D also suppressed DC maturation in vivo.

It has been reported that $C$. spinosa contained lots of components and showed various pharmacological activities including anti-inflammatory [6]. Several studies have been shown that flavonoids of C. spinosa are contributed to the anti-inflammatory activity $[14,16]$. DCs are the key regulators for the homeostasis of immune system and their changes in distribution, function, and cytokine production are closely correlated with autoimmune and inflammatory disorders [43,44]. Here, we found that CSEs contained same concentration of flavonoids exhibited different effects on DC maturation. CSEM and CSE2 promote DC maturation but CSE3 did not. Moreover, CSE3 suppressed DC maturation and pro-inflammatory cytokine production including IL-12, IL-6, IL-1 $\beta$, and TNF- $\alpha$ induced by LPS. The results indicated that CSEs might contain different components that resulted in different effects on DC maturation. The flavonoids in CSE3 might be the critical components that caused the anti-inflammatory activity to suppress cytokine production of DCs induced by LPS. Therefore, other components in CSEM and CSE2 or different flavonoids with them in CSE3 might promote DC maturation.

Some polysaccharides of herbal medicine could promote DC maturation and cytokine production [39-42]. We observed that CSEs contained high levels of polysaccharides. We used the same concentrations of polysaccharides from CSEs to treat DCs and found that CSEM had highest stimulatory activity to promote DC maturation, followed by CSE2. However, CSE3 did not promote DC maturation. The ratios of polysaccharides/flavonoids are 376, 85, and 368 in CSE2D, CSEMD, and CSE3D (Table 1). These results suggested that polysaccharides might not be the active components to promote DC maturation or different polysaccharides in CSEs had different effects on DC maturation. Bilen et al. [33] reported that caper methanolic extract stimulates innate immunity in rainbow trout, which elevated the levels of TNF- $\alpha$, IL-12p40, and IL-10 and increased phagocytic activity. In a future study, we will purify the active components that suppress or enhance DC maturation.

Taken together, CSE3 could suppress DC maturation and cytokine production induced by LPS both in vitro and in vivo, suggesting that CSE3 might be used as anti-inflammatory agent to treat arthritic and allergic diseases.

\section{Materials and Methods}

\subsection{Preparation of C. spinosa Ethanol Extracts}

The C. spinosa ethanol extracts were prepared using the procedures that were shown in Figure 1. Generally, the dried fruit of C. spinosa was crushed into powder. $400 \mathrm{~g}$ of powder was extracted using $2.4 \mathrm{~L}$ of absolute ethanol at $60{ }^{\circ} \mathrm{C}$ three times $(2 \mathrm{~h} /$ time). The extracts were collected, filtrated, and concentrated by a rotary evaporator. For CSE3 and CSEM, the ethanol extract was concentrated to $100 \mathrm{~mL}, 400 \mathrm{~mL}$ of distilled water was added and then extracted with petroleum ether three times. The extract was divided into three layers when it was extracted with petroleum ether. On the one 
hand, the middle layer was isolated and lyophilized and named as CSEM. On the other hand, the extract was treated with $\mathrm{NaCl}$ until it was divided into two layers. The water fraction was isolated and extracted with ethyl acetate three times, then the water fraction was concentrated by a rotary evaporator. During evaporation, crystallized $\mathrm{NaCl}$ was timely removed. Finally, this fraction was lyophilized and named as CSE3. For CSE2, the ethanol extract was concentrated to ethanol-free, $400 \mathrm{~mL}$ of distilled water was added and then extracted with petroleum ether three times. The extract was divided into two layers. The water fraction was isolated and extracted with ethyl acetate three times, then the water fraction was lyophilized and named CSE2. These CSEs were dissolved in distilled water or DMSO (Sigma-Aldrich, St. Louis, MO, USA) at $200 \mathrm{mg} / \mathrm{mL}$ and named CSE2W, CSEMW, and CSE3W; or CSE2D, CSEMD, and CSE3D, respectively.

The contents of flavonoids and polysaccharides were determined by AlCl3-KAC and anthrone-sulfuric acid method, respectively.

\subsection{Mice and Injection}

BALB/c mice were, aged 6-8 weeks, purchased from the Beijing laboratory animal research center (Beijing, China). Mice were housed in a temperature-controlled, light-cycled animal facility of Xinjiang University. All animal experiments were approved by the Committee on the Ethics of Animal Experiments of Xinjiang Key Laboratory of Biological Resources and Genetic Engineering and carried out under the guidelines of the Animal Care and Use Committee of College of Life Science and Technology, Xinjiang University.

Mice (3 mice/group) were injected with different concentrations $(0.4,0.8$, and $1.6 \mu \mathrm{g} / \mathrm{mouse})$ of flavonoids in CSE3D or LPS (100 ng/mouse) by both sides of footpads ( $25 \mu \mathrm{L} /$ footpad). After $24 \mathrm{~h}$, popliteal lymph nodes were isolated to analyze DC maturation by flow cytometry.

\subsection{The Generation of Dendritic Cell and CSE Treatment}

Immature DCs were induced from bone marrow cells of BALB/c mice in the presence of $20 \mathrm{ng} / \mathrm{mL}$ of GM-CSF (Peprotech) according to the previous protocol [45]. These cells were collected on day 7 and treated with different concentrations of CSEs in the absence or presence of $20 \mathrm{ng} / \mathrm{mL}$ LPS (Sigma-Aldrich). LPS treatment was used as a positive control.

\subsection{Enzyme-Linked Immunosorbent Assay (ELISA)}

Upon CSEs or LPS treatment, the levels of IL-12p40, IL-10, IL-6, IL-1 $\beta$, and TNF- $\alpha$ in supernatants of the above samples were measured by ELISA kit according to the manufacturer's instruction (Elabscience, Wuhan, China). The OD values at $450 \mathrm{~nm}$ were obtained using an ELISA plate reader (Bio-Rad, Hercules, CA, USA) and the concentrations of cytokines were calculated according to the standard curve.

\subsection{Flow Cytometry}

Annexin V/PI staining kit (Shanghai Yeasen Biotechnology Co., Ltd., Shanghai, China) was used to detect the viability of DCs upon CSE treatment and performed according to the manufacturer's instruction. For analysis of DC maturation, cell surface staining was performed using the mAbs (BD Biosciences, San Jose, CA, USA): PE-CD11c, APC-CD40, FITC-CD80, APC-CD11c, FITC-CD86, or PE-MHC II. All samples were collected on FACSCalibur (BD Biosciences) and the data were analyzed by the FlowJo platform (Tree Star, Inc., Ashland, OR, USA). CD11 $\mathrm{c}^{+}$cells were gated to analyze the expressions of CD40, CD80, CD86, and MHC II on DCs in all experiments.

\subsection{Statistical Analysis}

Statistical analysis was done by one-way analysis of variance (ANOVA). $p<0.05$ was considered to be statistically significant. 
Acknowledgments: This work was supported by the Chinese National Natural Science Foundation Grant (31460241) to J.L.

Author Contributions: Azeguli Hamuti, Jinyu Li, Fangfang Zhou and Adila Aipire performed the experiments; Ji Ma, Jianhua Yang and Jinyao Li designed the experiments; Jinyao Li analyzed the data and wrote the paper.

Conflicts of Interest: The authors declare no conflict of interest.

\section{References}

1. Li, J.; Li, J.; Zhang, F. The immunoregulatory effects of Chinese herbal medicine on the maturation and function of dendritic cells. J. Ethnopharmacol. 2015, 171, 184-195. [CrossRef] [PubMed]

2. Li, J.; Zhang, F.; Li, J. The immunoregulatory effects of traditional Chinese medicine on treatment of asthma or asthmatic inflammation. Am. J. Chin. Med. 2015, 43, 1059-1081. [CrossRef] [PubMed]

3. Chen, X.; Yang, L.; Howard, O.M.; Oppenheim, J.J. Dendritic cells as a pharmacological target of traditional Chinese medicine. Cell. Mol. Immunol. 2006, 3, 401-410. [PubMed]

4. Huseini, H.F.; Hasani-Rnjbar, S.; Nayebi, N.; Heshmat, R.; Sigaroodi, F.K.; Ahvazi, M.; Alaei, B.A.; Kianbakht, S. Capparis spinosa L. (Caper) fruit extract in treatment of type 2 diabetic patients: A randomized double-blind placebo-controlled clinical trial. Complement. Ther. Med. 2013, 21, 447-452. [CrossRef] [PubMed]

5. Sher, H.; Alyemeni, M.N. Ethnobotanical and pharmaceutical evaluation of Capparis spinosa L., validity of local folk and Unani system of medicine. J. Med. Plants Res. 2010, 4, 1751-1756.

6. Nabavi, S.F.; Maggi, F.; Daglia, M.; Habtemariam, S.; Rastrelli, L.; Nabavi, S.M. Pharmacological Effects of Capparis spinosa L. Phytother. Res. 2016, 30, 1733-1744. [CrossRef] [PubMed]

7. Tlili, N.; Elfalleh, W.; Saadaoui, E.; Khaldi, A.; Triki, S.; Nasri, N. The caper (Capparis L.): ethnopharmacology, phytochemical and pharmacological properties. Fitoterapia 2011, 82, 93-101. [CrossRef] [PubMed]

8. Ao, M.; Gao, Y.; Yu, L. Advances in studies on constituents and their pharmacological activities of Capparis spinosa. Chin. Tradit. Herb. Drugs 2007, 38, 463-467.

9. Lam, S.K.; Han, Q.F.; Ng, T.B. Isolation and characterization of a lectin with potentially exploitable activities from caper (Capparis spinosa) seeds. Biosci. Rep. 2009, 29, 293-299. [CrossRef] [PubMed]

10. Sharaf, M.; El-Ansari, M.A.; Saleh, N.A.M. Quercetin triglyco-side from Capparis spinosa. Fitoterapia 2000, 71, 46-49. [CrossRef]

11. Rodrigo, M.; Lazaro, M.J.; Alvarruiz, A.; Giner, V. Composition of capers (Capparis spinosa): Influence of cultivar, size and harvest date. J. Food Sci. 1992, 57, 1152-1154. [CrossRef]

12. Khatib, M.; Pieraccini, G.; Innocenti, M.; Melani, F.; Mulinacci, N. An insight on the alkaloid content of Capparis spinosa L. root by HPLC-DAD-MS, MS/MS and (1)H qNMR. J. Pharm. Biomed. Anal. 2016, 123, 53-62. [CrossRef] [PubMed]

13. Zhou, H.; Jian, R.; Kang, J.; Huang, X.; Li, Y.; Zhuang, C.; Yang, F.; Zhang, L.; Fan, X.; Wu, T.; et al. Anti-inflammatory effects of caper (Capparis spinosa L.) fruit aqueous extract and the isolation of main phytochemicals. J. Agric. Food Chem. 2010, 58, 12717-12721. [CrossRef] [PubMed]

14. Zhou, H.F.; Xie, C.; Jian, R.; Kang, J.; Li, Y.; Zhuang, C.L.; Yang, F.; Zhang, L.L.; Lai, L.; Wu, T.; et al. Biflavonoids from Caper (Capparis spinosa L.) fruits and their effects in inhibiting NF-kappa B activation. J. Agric. Food Chem. 2011, 59, 3060-3065. [CrossRef] [PubMed]

15. Moutia, M.; El Azhary, K.; Elouaddari, A.; Al Jahid, A.; Jamal Eddine, J.; Seghrouchni, F.; Habti, N.; Badou, A. Capparis spinosa L. promotes anti-inflammatory response in vitro through the control of cytokine gene expression in human peripheral blood mononuclear cells. BMC Immunol. 2016, 17, 26. [CrossRef] [PubMed]

16. Mohebali, N.; Shahzadeh Fazeli, S.A.; Ghafoori, H.; Farahmand, Z.; MohammadKhani, E.; Vakhshiteh, F.; Ghamarian, A.; Farhangniya, M.; Sanati, M.H. Effect of flavonoids rich extract of Capparis spinosa on inflammatory involved genes in amyloid-beta peptide injected rat model of Alzheimer's disease. Nutr. Neurosci. 2016, 1-8. [CrossRef] [PubMed]

17. Lam, S.K.; Ng, T.B. A protein with antiproliferative, antifungal and HIV-1 reverse transcriptase inhibitory activities from caper (Capparis spinosa) seeds. Phytomedicine 2009, 16, 444-450. [CrossRef] [PubMed]

18. Arena, A.; Bisignano, G.; Pavone, B.; Tomaino, A.; Bonina, F.P.; Saija, A.; Cristani, M.; D’Arrigo, M.; Trombetta, D. Antiviral and immunomodulatory effect of a lyophilized extract of Capparis spinosa L. buds. Phytother. Res. 2008, 22, 313-317. [CrossRef] [PubMed] 
19. Trombetta, D.; Occhiuto, F.; Perri, D.; Puglia, C.; Santagati, N.A.; de Pasquale, A.; Saija, A.; Bonina, F. Antiallergic and antihistaminic effect of two extracts of Capparis spinosa L. flowering buds. Phytother. Res. 2005, 19, 29-33. [CrossRef] [PubMed]

20. Feng, X.; Lu, J.; Xin, H.; Zhang, L.; Wang, Y.; Tang, K. Anti-arthritic active fraction of Capparis spinosa L. fruits and its chemical constituents. Yakugaku Zasshi 2011, 131, 423-429. [CrossRef] [PubMed]

21. Ji, Y.B.; Yu, L. N-butanol extract of Capparis spinosa L. induces apoptosis primarily through a mitochondrial pathway involving mPTP open, cytochrome C release and caspase activation. Asian Pac. J. Cancer Prev. 2014, 15, 9153-9157. [CrossRef] [PubMed]

22. Tesoriere, L.; Butera, D.; Gentile, C.; Livrea, M.A. Bioactive components of caper (Capparis spinosa L.) from Sicily and antioxidant effects in a red meat simulated gastric digestion. J. Agric. Food Chem. 2007, 55, 8465-8471. [CrossRef] [PubMed]

23. Cao, Y.L.; Li, X.; Zheng, M. Capparis spinosa protects against oxidative stress in systemic sclerosis dermal fibroblasts. Arch. Dermatol. Res. 2010, 302, 349-355. [CrossRef] [PubMed]

24. Germanò, M.P.; de Pasquale, R.; D'Angelo, V.; Catania, S.; Silvari, V.; Costa, C. Evaluation of extracts and isolated fraction from Capparis spinosa L. buds as an antioxidant source. J. Agric. Food Chem. 2002, 50, 1168-1171. [CrossRef] [PubMed]

25. Siracusa, L.; Kulisic-Bilusic, T.; Politeo, O.; Krause, I.; Dejanovic, B.; Ruberto, G. Phenolic composition and antioxidant activity of aqueous infusions from Capparis spinosa L. and Crithmum maritimum L. before and after submission to a two-step in vitro digestion model. J. Agric. Food Chem. 2011, 59, 12453-12459. [CrossRef] [PubMed]

26. Mansour, R.B.; Jilani, I.B.; Bouaziz, M.; Gargouri, B.; Elloumi, N.; Attia, H.; Ghrabi-Gammar, Z.; Lassoued, S. Phenolic contents and antioxidant activity of ethanolic extract of Capparis spinosa. Cytotechnology 2016, 68, 135-142. [CrossRef] [PubMed]

27. Maresca, M.; Micheli, L.; Di Cesare Mannelli, L.; Tenci, B.; Innocenti, M.; Khatib, M.; Mulinacci, N.; Ghelardini, C. Acute effect of Capparis spinosa root extracts on rat articular pain. J. Ethnopharmacol. 2016, 193, 456-465. [CrossRef] [PubMed]

28. Arslan, R.; Bektas, N.; Ozturk, Y. Antinociceptive activity of methanol extract of fruits of Capparis ovata in mice. J. Ethnopharmacol. 2010, 131, 28-32. [CrossRef] [PubMed]

29. Kazemian, M.; Abad, M.; Haeri, M.R.; Ebrahimi, M.; Heidari, R. Anti-diabetic effect of Capparis spinosa L. root extract in diabetic rats. Avicenna J. Phytomed. 2015, 5, 325-332. [PubMed]

30. Gadgoli, C.; Mishra, S.H. Antihepatotoxic activity of p-methoxy benzoic acid from Capparis spinosa. J. Ethnopharmacol. 1999, 66, 187-192. [CrossRef]

31. Jalali, M.T.; Mohammadtaghvaei, N.; Larky, D.A. Investigating the effects of Capparis spinosa on hepatic gluconeogenesis and lipid content in streptozotocin-induced diabetic rats. Biomed. Pharmacother. 2016, 84, 1243-1248. [CrossRef] [PubMed]

32. Eddouks, M.; Lemhadri, A.; Michel, J.B. Hypolipidemic activity of aqueous extract of Capparis spinosa L. in normal and diabetic rats. J. Ethnopharmacol. 2005, 98, 345-350. [CrossRef] [PubMed]

33. Bilen, S.; Altunoglu, Y.C.; Ulu, F.; Biswas, G. Innate immune and growth promoting responses to caper (Capparis spinosa) extract in rainbow trout (Oncorhynchus mykiss). Fish Shellfish Immunol. 2016, 57, $206-212$. [CrossRef] [PubMed]

34. Garg, R.; Shrivastava, P.; van Drunen Littel-van den Hurk, S. The role of dendritic cells in innate and adaptive immunity to respiratory syncytial virus, and implications for vaccine development. Expert Rev. Vaccines 2012, 11, 1441-1457. [CrossRef] [PubMed]

35. Kalinski, P. Dendritic cells in immunotherapy of established cancer: Roles of signals 1, 2, 3 and 4. Curr. Opin. Investig. Drugs 2009, 10, 526-535. [PubMed]

36. Mei, L. The Preparation of Capparis spinosa L. Ointment on Anti-Rheumatoid Arthritis. Master's Thesis, HuaZhong University of Science and Technology, Wuhan, China, 2012.

37. Agbo, M.O.; Uzor, P.F.; Akazie-Nneji, U.N.; Eze-Odurukwe, C.U.; Ogbatue, U.B.; Mbaoji, E.C. Antioxidant, total phenolic and flavonoid content of selected Nigerian medicinal plants. Dhaka Univ. J. Pharm. Sci. 2015, 14, 35-41. [CrossRef]

38. Lin, X.; Wang, S.; Jiang, Y.; Wang, Z.J.; Sun, G.L.; Xu, D.S.; Feng, Y.; Shen, L. Poly(ethylene glycol)-Radix Ophiopogonis polysaccharide conjugates: Preparation, characterization, pharmacokinetics and in vitro bioactivity. Eur. J. Pharm. Biopharm. 2010, 76, 230-237. [CrossRef] [PubMed] 
39. Du, X.; Zhao, B.; Li, J.; Cao, X.; Diao, M.; Feng, H.; Chen, X.; Chen, Z.; Zeng, X. Astragalus polysaccharides enhance immune responses of HBV DNA vaccination via promoting the dendritic cell maturation and suppressing Treg frequency in mice. Int. Immunopharmacol. 2012, 14, 463-470. [CrossRef] [PubMed]

40. Hsu, H.Y.; Hua, K.F.; Lin, C.C.; Lin, C.H.; Hsu, J.; Wong, C.H. Extract of Reishi polysaccharides induces cytokine expression via TLR4-modulated protein kinase signaling pathways. J. Immunol. 2004, 173, 5989-5999. [CrossRef] [PubMed]

41. Li, X.; Xu, W.; Chen, J. Polysaccharide purified from Polyporus umbellatus (Per) Fr induces the activation and maturation of murine bone-derived dendritic cells via toll-like receptor 4. Cell. Immunol. 2010, 265, 50-56. [CrossRef] [PubMed]

42. Lin, Y.L.; Liang, Y.C.; Lee, S.S.; Chiang, B.L. Polysaccharide purified from Ganoderma lucidum induced activation and maturation of human monocyte-derived dendritic cells by the NF-kappaB and p38 mitogen-activated protein kinase pathways. J. Leukoc. Biol. 2005, 78, 533-543. [CrossRef] [PubMed]

43. Coutant, F.; Miossec, P. Altered dendritic cell functions in autoimmune diseases: distinct and overlapping profiles. Nat. Rev. Rheumatol. 2016, 12, 703-715. [CrossRef] [PubMed]

44. Suryawanshi, A.; Tadagavadi, R.K.; Swafford, D.; Manicassamy, S. Modulation of Inflammatory Responses by Wnt/ $\beta$-Catenin Signaling in Dendritic Cells: A Novel Immunotherapy Target for Autoimmunity and Cancer. Front. Immunol. 2016, 7, 460. [CrossRef] [PubMed]

45. Li, J.; Wang, X.; Wang, W.; Luo, J.; Aipire, A.; Li, J.; Zhang, F. Pleurotus ferulae water extract enhances the maturation and function of murine bone marrow-derived dendritic cells through TLR4 signaling pathway. Vaccine 2015, 33, 1923-1933. [CrossRef] [PubMed]

Sample Availability: Samples of the compounds are available from the authors.

(c) 2017 by the authors; licensee MDPI, Basel, Switzerland. This article is an open access article distributed under the terms and conditions of the Creative Commons Attribution (CC-BY) license (http://creativecommons.org/licenses/by/4.0/). 